Chinese Journal of Organic Chemistry

\title{
雄甾-3,17-二醇衍生物的分子结构研究
}

\author{
方明锋*, $a$ 储刚 $b$ 李 歆 $a$ 陆国元*, $b, c$ \\ ( ${ }^{a}$ 南京林业大学化学工程学院 南京 210037) \\ ( ${ }^{b}$ 南京圣和药业公司 南京 210038) \\ ( ${ }^{c}$ 南京大学化学化工学院 南京 210093)
}

\begin{abstract}
摘要 罗库溴铵(Rocuronium Bromide)是新的手术前的甾醇类肌松药, 其安全性和有效性已得到临床研究和应用的证 实. 雄甾-3,17-二醇(1)是合成罗库溴铵的关键中间体. 用嗍氢化钠还原 $(2 \alpha, 3 \alpha, 5 \alpha, 16 \beta)$-2,3-环氧基-16-(1-吡咯)-17-氧代雄 甾烷(2), 然后在吗啉和水中回流使环氧开环得到雄甾-3,17-二醇(1). 高分辨 TOF-MS 质谱和 ${ }^{1} \mathrm{H} \mathrm{NMR},{ }^{13} \mathrm{C} \mathrm{NMR},{ }^{1} \mathrm{H}-{ }^{1} \mathrm{H}$ COSY, HSQC, DEPT, HMBC, 2D NOESY 等核磁共振谱表征确认其分子构造, 并且制备和测定了雄甾-3,17-二醇 1 的单 晶结构, 确认了它的立体构型. 雄甾-3,17-二醇(1)的样品的 $\mathrm{X}$ 粉末衍射分析研究表明其衍射谱与根据单晶数据计算的 理论谱一致, 因此, 合成的雄甾-3,17-二醇(1)的分子结构是 $(2 \beta, 3 \alpha, 5 \alpha, 16 \beta, 17 \beta)$-2-(4-吗㕲基)-16-(1-吡咯)雄甾-3,17-二醇, 其立体构型与罗库溴铵的立体构型一致.
\end{abstract}

关键词 雄甾二醇; 罗库澳铵; 核磁共振谱; 立体构型; 晶体结构

\section{Studies of Molecular Structures of 3,17-Androstandiol Derivative}

\author{
Fang, Mingfeng ${ }^{*, a} \quad$ Chu, Gang ${ }^{b} \quad \mathrm{Li}, \mathrm{Xin}^{a} \quad$ Lu, Guo-Yuan ${ }^{*, c, b}$ \\ ( ${ }^{a}$ College of Chemical Engineering, Nanjing Forestry University, Nanjing 210037) \\ ( ${ }^{b}$ Sanhome Pharmaceutical Co., Ltd., Nanjing 210038) \\ ( ${ }^{c}$ Department of Chemistry, Nanjing University, Nanjing 210093)
}

\begin{abstract}
Rocuronium bromide is a new neuromuscular blocking agent in clinical intervention. Its potency, specificity of action and little side effect have been confirmed. The 3,17-androstandiol (1) is a key intermediate for the preparation of rocuronium bromide. In this paper, The 3,17-androstandiol (1) was synthesized from $(2 \alpha, 3 \alpha, 5 \alpha, 16 \beta)$-2,3-epoxy-16(1-pyrrolidinyl)-17-oxoandrostane by a reduction with sodium borohydride in methanol and then an epoxy ring opening reaction in morpholine and water. The molecular constitution was confirmed by ${ }^{1} \mathrm{H} N M R,{ }^{13} \mathrm{C} N M R,{ }^{1} \mathrm{H}-{ }^{1} \mathrm{H}$ COSY, DEPT, HSQC,HMBC, 2D NOESY and TOF-MS spectra. The single crystal of 3,17-androstandiol (1) was prepared by crystallized from 2-MeTHF and then was subjected to X-ray crystallography analysis that confirmed the stereo configuration of compound 1. And studies of X-ray powder diffractive analysis indicate that the diffractive spectrum of the sample compound $\mathbf{1}$ is consistent with the diffractive spectrum calculated on the crystal data. Therefore, the compound is $(2 \beta, 3 \alpha, 5 \alpha, 16 \beta, 17 \beta)$ 2-(4-morpholinyl)-16-(1-pyrrolidinyl)androstan-3,17-diol and its stereochemistry is accordant with that of rocuronium bromide.
\end{abstract}

Keywords androstandiol; rocuronium bromide; NMR spectra; configuration; crystal structure

罗库溴铵 (Rocuronium Bromide, 1-[(2 $\beta, 3 \alpha, 5 \alpha, 16 \beta$, $17 \beta$ )-17-乙酰氧基-3-羊基-2-(4-吗啉基)雄甾烷-16-基]1-(2-丙烯基)吡咯烷溴化物)是甾醇类非去极性化肌松 药. 罗库溴铵具有起效快、体内无蓄积、没有任何不良 反应, 其安全性和有效性已得到临床研究和应用的证
实. 因此罗库溴铵已被广泛用作外科手术前麻醉肌松 药 ${ }^{[1 \sim 4]}$.

文献 5 ～13]报道罗库溴铵的制备有多条合成路线， 所有路线都是以雄表酮为起始原料经多步合成得到 (2 $\beta, 3 \alpha, 5 \alpha, 16 \beta, 17 \beta)$-2-(4- 吗啉基)-16-(1-吡咯)雄甾-3,17-

\footnotetext{
*E-mail: hgfmf@njfu.edu.cn

Received April 17, 2014; revised June 1, 2014; published online June 18, 2014.

Project supported by the National Natural Science Foundation of China (No. 31200451), the Natural Science Foundation of Jiangsu Province (No. BK2012821) and the Foundation of Priority Academic Program Development (PAPD) of Jiangsu Higher Education Institutions.

国家自然科学青年基金(No. 31200451)、江苏省自然科学基金(No. BK2012821)、江苏高校优势学科建设工程资助项目.
} 
二醇(简写作雄甾二醇中间体 $\mathbf{1}$ ), 然后雄甾二醇中间体 $\mathbf{1}$ 经 17-差基乙酰化和吡咯环氮原子上烯丙基化得到罗库 溴铵(图 1). 因此雄甾二醇是合成罗库溴铵的关键中间 体. 该中间体的合成中手性结构的确认和可能的手性杂 质的控制对罗库溴铵的质量至关重要. 但是文献迄今没 有报道雄甾二醇的结构论证. 因此本文用核磁共振光谱 和 $\mathrm{X}$ 单晶衍射的方法全面论证和解析合成得到雄甾二 醇中间体 1 的立体结构.

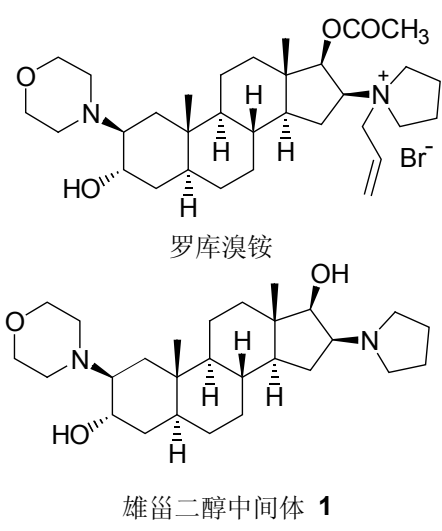

图 1 罗库溴铵和雄甾二醇中间体 $\mathbf{1}$ 的结构

Figure 1 Structures of rocuronium bromide and androstandiol intermediate

\section{1 结果与讨论}

\section{1 雄甾二醇中间体 1 的合成}

雄甾二醇中间体 $\mathbf{1}$ 是合成罗库溴铵关键中间体. 本 文根据文献 $[11 ， 12]$ 稍加改进的方法合成(Scheme 1). (2 $\alpha, 3 \alpha, 5 \alpha, 16 \beta)$-2,3-环氧基-16-(1-吡咯)-17-氧代雄甾烷 (2)分子中 17 位羰基在二氯甲烷和甲醇混合溶剂经硼氢 化钠还原为羟基, 然后在吗啉和少量水中回流开环反应 生成化合物 1. 两步产率分别为 $60.1 \%$ 和 $55.2 \%$.

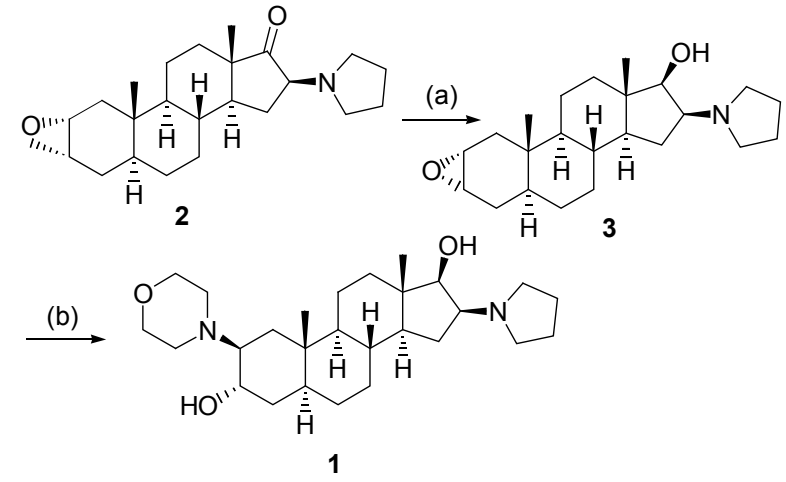

Reagents and conditions: (a) $\mathrm{NaBH}_{4}, \mathrm{CH}_{2} \mathrm{Cl}_{2} / \mathrm{CH}_{3} \mathrm{OH}, 0{ }^{\circ} \mathrm{C}, 6 \mathrm{~h}$; $60.1 \%$. (b) morpholine $/ \mathrm{H}_{2} \mathrm{O}$, refluxed, $48 \mathrm{~h} ; 55.2 \%$

Scheme 1

\section{2 雄甾二醇中间体 1 的核磁共振光谱解析}

雄甾二醇中间体 $\mathbf{1}$ 的核磁共振数据和归属见表 1 . 雄甾二醇中间体 1 分子中共有 27 个碳，由于分子吡咯环 的 $\mathrm{C}_{25}$ 和 $\mathrm{C}_{26}, \mathrm{C}_{24}$ 和 $\mathrm{C}_{27}$, 吗啉环的 $\mathrm{C}_{21}$ 和 $\mathrm{C}_{22}, \mathrm{C}_{20}$ 和 $\mathrm{C}_{23}$ 是等价碳, 所以 ${ }^{13} \mathrm{C} N M R$ 谱显示有 23 个碳信号峰, 与 化合物的不等价碳的数目一致. DEPT 谱和 HSQC 谱显 示有 2 个伯碳, 11 个仲碳, 8 个叔碳, 2 个季碳信号峰, 因 此碳的种类和数目和化合物 $\mathbf{1}$ 相符合. HSQC 谱中, 伯碳 信号 $\delta 12.9,16.7$ 相应的氢谱化学位移分别是 $\delta 0.70(\mathrm{~s}$, $3 \mathrm{H}), 0.88(\mathrm{~s}, 3 \mathrm{H})$, 显然它们可归属为甾环上两个角甲基 信号峰. HSQC 谱中仲碳信号 $\delta 49.1$ 和 67.5 相应的氢谱 化学位移分别是 $\delta 2.43 \sim 2.45(\mathrm{~m}, 2 \mathrm{H})$ 和 $2.63 \sim 2.67(\mathrm{~m}$, 2H), 3.69 3.74 (m, 4H), ${ }^{1} \mathrm{H}-{ }^{1} \mathrm{H}$ COSY 谱显示它们互相 偶合，因此可以归属为吗啉环上 4 个亚甲基的信号峰. HSQC 谱中仲碳信号 $\delta 23.4$ 和 53.0 相应的氢谱化学位 移分别是 $\delta 1.74 \sim 1.77(\mathrm{~m}, 4 \mathrm{H}), 2.54 \sim 2.56(\mathrm{~m}, 2 \mathrm{H})$ 和 $2.76 \sim 2.79(\mathrm{~m}, 2 \mathrm{H}),{ }^{1} \mathrm{H}-{ }^{1} \mathrm{H}$ COSY 谱显示它们互相偶合, 因此可以归属为吡咯环上 4 个亚甲基的信号峰. HSQC 谱中叔 C 信号 $\delta 65.2$ 和 63.9 相应的氢谱化学位移分别 是 $\delta 2.52 \sim 2.53(\mathrm{~m}, 1 \mathrm{H})$ 和 $3.88 \sim 3.90(\mathrm{~m}, 1 \mathrm{H}),{ }^{1} \mathrm{H}-{ }^{1} \mathrm{H}$ COSY 谱显示它们互相偶合. 叔 C 信号 $\delta 63.4$ 和 79.2 相 应的氢谱化学位移分别是 $\delta 2.93 \sim 2.95(\mathrm{~m}, 1 \mathrm{H})$ 和 $3.38 \sim 3.40(\mathrm{~m}, 1 \mathrm{H}),{ }^{1} \mathrm{H}-{ }^{1} \mathrm{H}$ COSY 谱显示它们互相偶合, 因此它们分别是与吗啉环氮、羟基氧，四氢吡咯氮、羟 基氧相连的叔碳叔氢的信号峰. $\delta 21.0,28.2,29.3,31.8$, $32.7,34.4,38.5$ 为雄甾烷母核上 7 个亚甲基的仲碳信号, $\delta 34.7,38.6,48.5,56.1$ 为雄甾烷母核上无杂原子相连的 叔碳信号峰, $\delta 36.0$ 和 43.6 为雄甾烷母核上的两个季碳 信号峰. 根据 HMBC 谱可以进一步确认甾环上仲碳和 季碳的归属(表 1). 从 2D NOESY 谱可以推断该化合物 的构型. 甾体母核中角甲基 $\mathrm{C}_{18}$ 和 $\mathrm{C}_{19}$ 均为 $\beta$ 构型. NOESY 谱中, $\mathrm{H}_{3}$ 与 $\mathrm{H}_{19}$ 相关, 可知 $\mathrm{H}_{3}$ 为 $\beta$ 构型, 即 $\mathrm{C}_{3}$ 上的羟基为 $\alpha$ 构型; $\mathrm{H}_{3}$ 与吗啉环的 $\mathrm{H}_{20}, \mathrm{H}_{23}$ 相关, 可知 $\mathrm{H}_{2}$ 为 $\alpha$ 构型, 即吗啉环氮原子与甾环 $\mathrm{C}_{2}$ 连接是 $\beta$ 构型. 同样，甾体母核中 $\mathrm{H}_{14}$ 为 $\alpha$ 构型, NOESY 谱中, $\mathrm{H}_{14}$ 与 $\mathrm{H}_{16}$ 相关, 可知 $\mathrm{H}_{16}$ 为 $\alpha$ 构型, 即四氮吡咯环氮原子与甾环 $\mathrm{C}_{16}$ 连接是 $\beta$ 构型; 并且 $\mathrm{H}_{16}$ 与 $\mathrm{H}_{17}$ 相关, 从而得出 $\mathrm{H}_{17}$ 为 $\alpha$ 构型, 即 $\mathrm{C}_{17}$ 上的羟基为 $\beta$ 构型. 综上所述核磁共振 数据与雄甾二醇中间体 $\mathbf{1}$ 的结构相吻合.

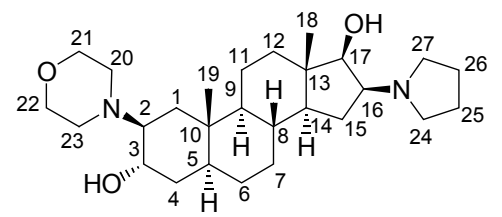


表 1 雄甾二醇中间体 $\mathbf{1}$ 的核磁共振光谱数据

Table 1 NMR data of androstandiol intermediate 1

\begin{tabular}{|c|c|c|c|c|c|}
\hline${ }^{13} \mathrm{C}$ NMR $\delta$ & 类型(DEPT) & 归属 & $\begin{array}{c}\text { 相应的 }{ }^{1} \mathrm{H} \text { NMR } \delta \\
\text { (HSQC) }\end{array}$ & $\begin{array}{l}{ }^{1} \mathrm{H}-{ }^{1} \mathrm{H} \text { 相关的 } \delta \\
\left({ }^{1} \mathrm{H}-{ }^{1} \mathrm{H} \text { COSY }\right)\end{array}$ & $\begin{array}{c}\text { 远程相关质子 } \delta \\
(\mathrm{HMBC})\end{array}$ \\
\hline 12.9 & 伯 C & $\mathrm{C}_{18}$ & $0.70(\mathrm{~s}, 3 \mathrm{H})$ & - & $1.11,1.91$ \\
\hline 16.7 & 伯 C & $\mathrm{C}_{19}$ & $0.88(\mathrm{~s}, 3 \mathrm{H})$ & - & 1.45 \\
\hline 21.0 & 仲 C & 甾环 $C_{11}$ & $\begin{array}{l}1.34 \sim 1.35(\mathrm{~m}, 1 \mathrm{H}) \\
1.53 \sim 1.55(\mathrm{~m}, 1 \mathrm{H})\end{array}$ & $\begin{array}{l}0.71,1.11 \\
0.71,1.11\end{array}$ & $0.71,1.11,1.91$ \\
\hline 23.4 & 仲 C & $\mathrm{C}_{25}, \mathrm{C}_{26}$ & $1.74 \sim 1.77(\mathrm{~m}, 4 \mathrm{H})$ & $2.54,2.76$ & $2.54,2.76$ \\
\hline 28.2 & 仲 C & 甾环 $\mathrm{C}_{6}$ & $\begin{array}{l}1.17 \sim 1.19(\mathrm{~m}, 1 \mathrm{H}) \\
1.39 \sim 1.41(\mathrm{~m}, 1 \mathrm{H})\end{array}$ & $\begin{array}{l}0.86,1.67 \\
0.86,1.67\end{array}$ & $1.56,1.67$ \\
\hline 29.3 & 仲 C & 甾环 $C_{15}$ & $\begin{array}{l}1.29 \sim 1.31(\mathrm{~m}, 1 \mathrm{H}) \\
1.81 \sim 1.82(\mathrm{~m}, 1 \mathrm{H})\end{array}$ & $\begin{array}{l}0.79,2.93 \\
0.79,2.93\end{array}$ & 0.79 \\
\hline 31.8 & 仲 C & 甾环 $C_{7}$ & $\begin{array}{l}0.86 \sim 0.87(\mathrm{~m}, 1 \mathrm{H}) \\
1.67 \sim 1.69(\mathrm{~m}, 1 \mathrm{H})\end{array}$ & $\begin{array}{l}1.17,1.39 \\
1.17,1.39\end{array}$ & $1.17,1.39$ \\
\hline 32.7 & 仲 C & 甾环 $C_{1}$ & $1.45 \sim 1.47(\mathrm{~m}, 2 \mathrm{H})$ & 2.52 & $0.88,2.52$ \\
\hline 34.4 & 仲 C & 甾环 $\mathrm{C}_{4}$ & $\begin{array}{l}1.48 \sim 1.49(\mathrm{~m}, 1 \mathrm{H}) \\
1.81 \sim 1.82(\mathrm{~m}, 1 \mathrm{H})\end{array}$ & $\begin{array}{l}1.56,3.88 \\
1.56,3.88\end{array}$ & $1.56,2.52$ \\
\hline 34.7 & 叔 C & 甾环 $\mathrm{C}_{8}$ & $1.35 \sim 1.36(\mathrm{~m}, 1 \mathrm{H})$ & $0.71,0.79$ & $0.71,0.86,1.67$ \\
\hline 36.0 & 季 C & 甾环 $C_{10}$ & - & - & 0.88 \\
\hline 38.5 & 仲 C & 甾环 $\mathrm{C}_{12}$ & $\begin{array}{l}1.11 \sim 1.12(\mathrm{~m}, 1 \mathrm{H}) \\
1.91 \sim 1.95(\mathrm{~m}, 1 \mathrm{H})\end{array}$ & $\begin{array}{l}1.34,1.53 \\
1.34,1.53\end{array}$ & $0.70,1.34,1.53$ \\
\hline 38.6 & 叔 C & 甾环 $C_{5}$ & $1.56 \sim 1.58(\mathrm{~m}, 1 \mathrm{H})$ & $1.17,1.39$ & $0.88,1.17,1.48$ \\
\hline 43.6 & 季 C & 甾环 $C_{13}$ & - & - & 0.70 \\
\hline 48.5 & 叔 C & 甾环 C 14 & $0.79 \sim 0.80(\mathrm{~m}, 1 \mathrm{H})$ & $1.29,1.81$ & $0.70,1.35,1.81$ \\
\hline 49.1 & 仲 C & $\mathrm{C}_{20}, \mathrm{C}_{23}$ & $\begin{array}{l}2.43 \sim 2.45(\mathrm{~m}, 2 \mathrm{H}) \\
2.63 \sim 2.67(\mathrm{~m}, 2 \mathrm{H})\end{array}$ & $\begin{array}{l}3.69 \\
3.69\end{array}$ & 3.69 \\
\hline 53.0 & 仲 C & $\mathrm{C}_{24}, \mathrm{C}_{27}$ & $\begin{array}{l}2.54 \sim 2.56(\mathrm{~m}, 2 \mathrm{H}) \\
2.76 \sim 2.79(\mathrm{~m}, 2 \mathrm{H})\end{array}$ & $\begin{array}{l}1.74 \\
1.74\end{array}$ & 1.74 \\
\hline 56.1 & 叔 C & 甾环 $C_{9}$ & $0.71 \sim 0.72(\mathrm{~m}, 1 \mathrm{H})$ & $1.35,1.53$ & $0.88,1.35,1.53$ \\
\hline 63.4 & 叔 C & 甾环 $\mathrm{C}_{16}$ & $2.93 \sim 2.95(\mathrm{~m}, 1 \mathrm{H})$ & $1.29,1.81,3.38$ & $1.29,1.81,3.38$ \\
\hline 63.9 & 叔 C & 甾环 $C_{3}$ & $3.88 \sim 3.90(\mathrm{~m}, 1 \mathrm{H})$ & $1.48,1.81,2.52$ & $1.48,1.81,2.52$ \\
\hline 65.2 & 叔 C & 甾环 $\mathrm{C}_{2}$ & $2.52 \sim 2.53(\mathrm{~m}, 1 \mathrm{H})$ & $1.45,3.88$ & $1.45,3.88$ \\
\hline 67.5 & 仲 C & $\mathrm{C}_{21}, \mathrm{C}_{22}$ & $3.69 \sim 3.74(\mathrm{~m}, 4 \mathrm{H})$ & $2.43,2.63$ & $2.43,2.63$ \\
\hline 79.2 & 叔 C & 甾环 $\mathrm{C}_{17}$ & $3.38 \sim 3.40(\mathrm{~m}, 1 \mathrm{H})$ & 2.93 & $0.70,2.93$ \\
\hline
\end{tabular}

\section{3 雄甾二醇中间体 1 的晶体结构}

雄甾二醇中间体 1 的 2-甲基四氢呋喃(2-MeTHF)溶 液在室温慢慢挥发从溶液得到 $(0.32 \mathrm{~mm} \times 0.30 \mathrm{~mm} \times$ $0.13 \mathrm{~mm})$ 块状单晶. 单晶衍射数据在 Bruker APEX DUO CCD 单晶衍射仪 $(\mathrm{Cu} \mathrm{K} \alpha, \lambda=1.54178 \AA)$ 采集. 单 晶结构和结构修正数据列于表 2 .

图 2 是单晶结构图. 可以确认与吗啉环氮原子相连 的叔碳 $\mathrm{C}_{2}$, 与四氢吡咯氮原子相连的叔碳 $\mathrm{C}_{16}$ 的绝对构 型都是 $S$-构型. 与羟基氧原子相连的甾环叔碳 $\mathrm{C}_{3}$ 和 $\mathrm{C}_{17}$ 的绝对构型分别是 $S$-构型和 $R$-构型. 即化合物 1 是 (2 $\beta, 3 \alpha, 5 \alpha, 16 \beta, 17 \beta)$-2-(4-吗啉基)-16-(1-吡咯)雄甾-3,17二醇.

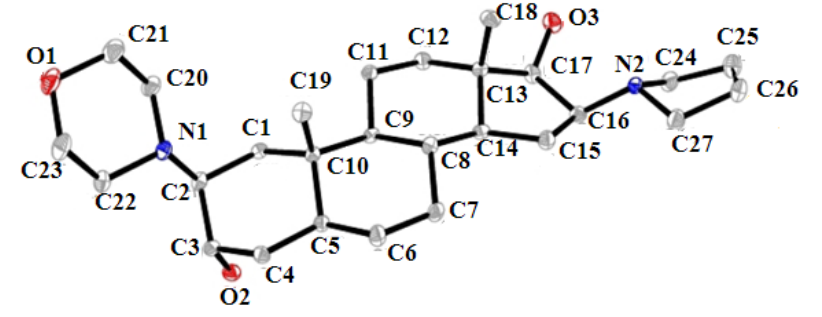

图 2 雄甾二醇中间体 $\mathbf{1}$ 的单晶结构图

Figure 2 Crystal data and structure of the androstandiol intermediate 1 
表 2 雄甾二醇中间体 $\mathbf{1}$ 的单晶结构和结构修正数据

Table 2 Crystal data and structure refinement for androstandiol intermediate 1

\begin{tabular}{ll}
\hline \multicolumn{1}{c}{ Empirical formula } & \multicolumn{1}{c}{$\mathrm{C}_{27} \mathrm{H}_{46} \mathrm{~N}_{2} \mathrm{O}_{3}$} \\
\hline Formula weight & 446.66 \\
Temperature & $173.15 \mathrm{~K}$ \\
Radiation type & $\mathrm{Cu} \mathrm{K \alpha}$ \\
Wavelength & $1.54178 \AA$ \\
Crystal system, & Monoclinic, $P 2_{1}$ \\
space group & $a=5.99500(10) \AA$ \\
Unit cell dimensions & $b=18.5747(4) \AA$ \\
& $c=11.4593(3) \AA$ \\
& $\alpha=90^{\circ}$ \\
& $\beta=95.7560(10)^{\circ}$ \\
& $\gamma=90^{\circ}$ \\
& $1269.62(5) \AA^{3}$ \\
Volume & $2,1.168 \mathrm{~kg} / \mathrm{m}^{3}$ \\
$Z$, Calculated density & $0.585 \mathrm{~mm}{ }^{-1}$ \\
Absorption coefficient & 492.0 \\
$F(000)$ & $0.32 \mathrm{~mm} \times 0.3 \mathrm{~mm} \times 0.13 \mathrm{~mm}$ \\
Crystal size & $3.88^{\circ}$ to $65.99^{\circ}$ \\
Theta range for data & \\
collection & $-7 \leqslant h \leqslant 6,-21 \leqslant k \leqslant 20$, \\
Limiting indices & $-13 \leqslant l \leqslant 13$ \\
Reflections collected/ & $6039 / 3320[R($ int $)=0.0475]$ \\
unique & $94.2 \%$ \\
Completeness & $3320 / 1 / 294$ \\
Refinement method & 1.053 \\
Data/restraints/parameters & $R_{1}=0.0756, w R_{2}=0.1849$ \\
Goodness-of-fit on $F^{2}$ & $0.27(17)$ \\
Final $R$ indices $[I>2 \sigma(I)]$ & 0.56 and $-0.53 \mathrm{e} \bullet \mathrm{A}^{-3}$ \\
Absolute structure Flack & \\
Largest diff. peak and hole & \\
\hline
\end{tabular}

图 3 是单晶的晶体堆积图. 雄甾二醇中间体 1 通过 分子间氢键 $\mathrm{O}(3)-\mathrm{H}(3) \cdots \mathrm{O}(2)(2.865 \AA)$ 和 $\mathrm{O}(2)-$ $\mathrm{H}(2) \cdots \mathrm{N}(2)(2.803 \AA)$ 形成链状结构.

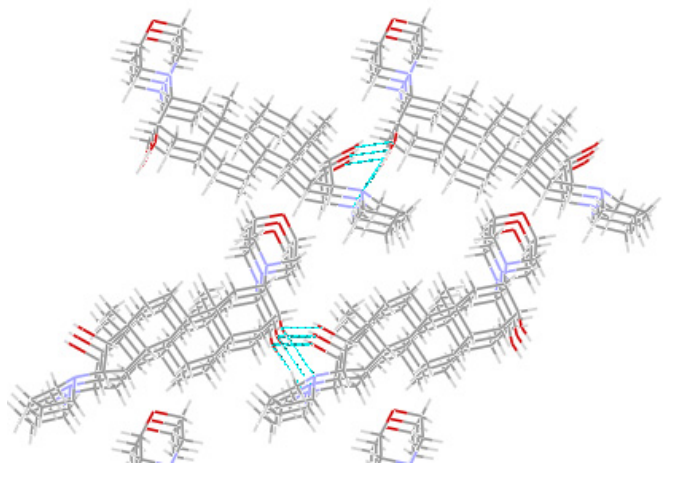

图 3 雄甾二醇中间体 $\mathbf{1}$ 的单晶的晶体堆积图

Figure 3 Crystal packing of the androstandiol intermediate 1
雄甾二醇中间体 1 的单晶数据 cif 文件(CCDC 996248) 已保存在剑桥大学晶体中心 [www.ccdc.cam.ac. uk/conts/retrieving.html (or from the Cambridge Crystallographic Data Centre, 12 Union Road, Cambridge CB2 1EZ, UK; Fax: + 44(0)1223-336033; Email: deposit@ ccdc.cam.ac.uk)].

\section{4 雄甾二醇中间体 1 的 X 粉末衍射谱}

雄甾二醇中间体 1 晶体的 $\mathrm{X}$ 射线粉末衍射谱用 EMPYREAN 型 $X$ 射线衍射仪连续扫描模式测定.扫描 范围 $2 \theta$ 为 $3^{\circ} \sim 40^{\circ}$. 图 3(a)是化合物 1 的 $X$ 射线粉末衍 射谱, 图 3(b)是根据化合物 1 的单晶结构数据计算得到 的理论衍射谱. 可以看到两者的主要峰的衍射角 $(2 \theta)$ 基 本相同，可以认为所合成得到的化合物 1 的全部晶体 (不是某个单晶)是纯粹的化合物 $(2 \beta, 3 \alpha, 5 \alpha, 16 \beta, 17 \beta)$ )-2-(4吗啉基)-16-(1-吡咯)雄甾-3,17-二醇.

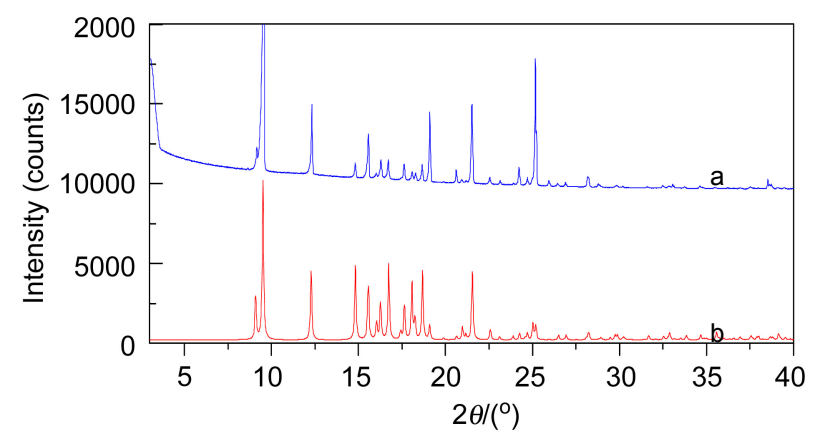

图 4 雄甾二醇中间体 $\mathbf{1}$ 的晶体的 X 粉末衍射图

Figure 4 XRPD spectra of androstandiol intermediate 1 crystal (a) Sample, (b) theoretic spectrum

\section{2 结论}

(2 $\alpha, 3 \alpha, 5 \alpha, 16 \beta)$-2,3-环氧基-16-(1-吡咯)-17-氧代雄甾 烷(2)经硼氢化钠还原, 然后在吗啉和水中回流使环氧 开环得到雄甾-3,17-二醇(1). 高分辨 TOF-MS 质谱和 ${ }^{1} \mathrm{H}$ NMR, ${ }^{13} \mathrm{C}$ NMR, ${ }^{1} \mathrm{H}^{-1} \mathrm{H}$ COSY, HSQC ,DEPT,HMBC, 2D NOESY 等核磁共振谱表征确认其分子构造. 并且制备 和测定了雄甾-3,17-二醇(1)的单晶结构, 确认了它的立 体构型. 雄甾-3,17-二醇(1)的样品的 X 粉末衍射分析研 究表明其衍射谱与根据单晶数据计算的理论谱一致. 因 此, 合成的雄甾 -3,17-二醇 (1) 的分子结构是 (2 $\beta, 3 \alpha, 5 \alpha, 16 \beta, 17 \beta)-2$-(4- 吗啉基)-16-(1-吡咯)雄甾-3,17二醇, 其立体构型与罗库溴铵的立体构型一致.

\section{3 实验部分}

\section{1 仪器与试剂}

熔点用 SGW X-4 显微熔点仪测定. ${ }^{1} \mathrm{H}$ NMR 谱和

${ }^{13} \mathrm{C}$ NMR 谱均用 Bruker AM $500 \mathrm{MHz}$ (Germany)核磁共 
振仪测定 $\left(\mathrm{CDCl}_{3}\right.$ 为溶剂, TMS 为内标). 高分辨质谱用 MAT 95 XP 质谱仪(LCQ, Finnigan)测定. 单晶结结构由 Bruker APEX DUO CCD 单晶衍射仪 $(\mathrm{Cu} \mathrm{K} \alpha, \lambda=1.54178$ $\AA$ )测定. $X$ 射线粉末衍射谱用 EMPYREAN 型 X 射线衍 射仪(Panalytical 公司)测定. 化合物 $\mathbf{2}$ 从济南高德医药科 技公司购买. 化合物 $\mathbf{3}$ 根据文献 $[11,12]$ 稍加改进的方法 合成. 试剂均为市售分析纯或化学纯试剂, 未进一步纯 化. 溶剂的干燥及无水处理按常规方法进行.

\section{$3.2(2 \alpha, 3 \alpha, 5 \alpha, 16 \beta, 17 \beta)-2,3$-环氧基-16-(1-吡咯)-17-} 羟基雄甾烷(3)的制备

将 $3.7 \mathrm{~g}(10 \mathrm{mmol})$ 化合物 $\mathbf{2}$ 溶解于 $30 \mathrm{~mL}$ 二氯甲烷 和 $20 \mathrm{~mL}$ 甲醇混合溶剂中, 冰浴冷却至 $0{ }^{\circ} \mathrm{C}$. 在搅拌下 分批加入 $3.0 \mathrm{~g}$ 嗍氢化钠. 然后在继续摚拌反应 $6 \mathrm{~h}$. 反 应结束后, 加入 $30 \mathrm{~mL}$ 水, 搅拌 $10 \mathrm{~min}$. 分出有机层, 无水硫酸镁干燥. 然后减压蒸去溶剂, 用丙酮重结晶得 到化合物 3 ( $2.6 \mathrm{~g}, 6.2 \mathrm{mmol}$ ), 白色固体, m.p. 168 170 ${ }^{\circ} \mathrm{C}$, 产率 60.1\%. ${ }^{1} \mathrm{H}$ NMR $\left(500 \mathrm{MHz}, \mathrm{CDCl}_{3}\right) \delta: 0.70$ (s, $3 \mathrm{H}), 0.71 \sim 0.73(\mathrm{~m}, 1 \mathrm{H}), 0.78 \sim 0.79(\mathrm{~m}, 1 \mathrm{H}), 0.85 \sim 0.86$ (m, $1 \mathrm{H}), 0.88(\mathrm{~s}, 3 \mathrm{H}), 1.11 \sim 1.17(\mathrm{~m}, 2 \mathrm{H}), 1.28 \sim 1.30(\mathrm{~m}$, $1 \mathrm{H}), 1.34 \sim 1.39(\mathrm{~m}, 3 \mathrm{H}), 1.44 \sim 1.47(\mathrm{~m}, 2 \mathrm{H}), 1.53 \sim 1.57$ $(\mathrm{m}, 2 \mathrm{H}), 1.67 \sim 1.69(\mathrm{~m}, 2 \mathrm{H}), 1.74 \sim 1.79(\mathrm{~m}, 4 \mathrm{H}), 1.81 \sim$ $1.83(\mathrm{~m}, 2 \mathrm{H}), 1.90 \sim 1.92(\mathrm{~m}, 1 \mathrm{H}), 2.52 \sim 2.58(\mathrm{~m}, 4 \mathrm{H})$, $2.92 \sim 2.95(\mathrm{~m}, 1 \mathrm{H}), 3.37 \sim 3.40(\mathrm{~m}, 1 \mathrm{H}), 3.78 \sim 3.88(\mathrm{~m}$, $2 \mathrm{H})$; MS (ESI) $m / z: 360.3[\mathrm{M}+\mathrm{H}]^{+}$.

\section{3 (2 $\beta, 3 \alpha, 5 \alpha, 16 \beta, 17 \beta)$-2-(4-吗啉基)-16-(1-吡咯)雄} 甾-3,17-二醇(1)的制备

将 $3.6 \mathrm{~g}(10 \mathrm{mmol})$ 化合物 $\mathbf{3}$ 溶解于 $40 \mathrm{~mL}$ 吗啉和 $4 \mathrm{~mL}$ 水中, 搅拌加热回流反应 $40 \mathrm{~h}$. 反应结束后, 减压 蒸去溶剂, 用丙酮重结晶得到化合物 $\mathbf{1}$ (2.48 g, 5.5 $\mathrm{mmol}$ ), 产率 $55.2 \%$, 白色固体. m.p. $212 \sim 216{ }^{\circ} \mathrm{C}$; $[\alpha]_{\mathrm{D}}^{20}=88.1\left(c 0.1, \mathrm{CHCl}_{3}\right)$ [文献值 ${ }^{[5]}$ m.p. $212 \sim 219{ }^{\circ} \mathrm{C}$; $\left.[\alpha]_{\mathrm{D}}^{20}=87.9\left(c 0.1, \mathrm{CHCl}_{3}\right)\right] .{ }^{1} \mathrm{H} \mathrm{NMR}$ 和 ${ }^{13} \mathrm{C} \mathrm{NMR}$ 光谱数 据见表 1. HRMS calcd for $\mathrm{C}_{27} \mathrm{H}_{47} \mathrm{~N}_{2} \mathrm{O}_{3}[\mathrm{M}+\mathrm{H}]^{+}$ 447.3508, found 447.3589,.

\section{4 雄甾二醇中间体 1 单晶的制备和结构测定}

将雄甾二醇中间体 1 溶解于 2-甲基四氢呋喃
(2-MeTHF) 中, 慢慢挥发从溶液中析出得到块状单晶 $(0.32 \mathrm{~mm} \times 0.3 \mathrm{~mm} \times 0.13 \mathrm{~mm})$. 单晶衍射数据在 Bruker APEX DUO CCD 单晶衍射仪 $(\mathrm{Cu} \mathrm{K \alpha}, \lambda=1.54178 \AA$ )采 集. Bruker SHELXS-97 程序解析单晶结构.

\section{5 雄甾二醇中间体 1 的 X 射线粉末衍射测定}

$X$ 射线粉末衍射(XRPD)使用 EMPYREAN 型 $X$ 射 线 $(\mathrm{Cu}, \mathrm{k} \alpha)$ 衍射仪(Panalytical 公司)测定. 采用单色器 (Focusing X-ray Mirror)连续扫描模式, 扫描范围 $3^{\circ} \sim$ $40^{\circ}(2 \theta)$, 扫描步长 $0.013^{\circ}(2 \theta)$, 扫描速度 $2.5\left(^{\circ}\right) / \mathrm{min}$.

辅助材料(Supporting Information) 化合物 $\mathbf{1}$ 的高分辨 质谱(HRMS)及 ${ }^{1} \mathrm{HNMR},{ }^{13} \mathrm{C} \mathrm{NMR},{ }^{1} \mathrm{H}-{ }^{1} \mathrm{H}$ COSY, HSQC, DEPT, HMBC, 2D NOESY 等核磁共振谱. 这些材料可 以免费从本刊网站(http://sioc-journal.cn/)上下载.

\section{References}

[1] Sparr, J.; Beaufort, M.; Fuchs-Buder, T. Drugs 2001, 61(7), 919.

[2] Lee, C. Pharmacol. Ther. 2003, 98(2), 143.

[3] European Pharmacopoeia Commission, European Pharmacopoeia 6.0, Strasbourg, 2008, pp. 2835 2837 .

[4] Zhang, Y. M.; Guo, J.; Xiong, T. Chin. J. Med. Chem. 2008, 18(6), 434 (in Chinese). (张永明, 郭佳, 熊涛, 中国药物化学杂志, 2008, 18(6), 434.)

[5] Savage, D. S.; Sleigh, T.; Carlyle, I. G. EP 287150, 1988 [Chem. Abstr. 1989, 110, 115184].

[6] Buckett, W. R.; Hewett, C. L.; Savage, D. S. J. Med. Chem. 1973, 16, 1116 .

[7] Yan, H.; Qiu, F.; Diao, Y. Chin. J. Pharm. 2011, 42(6), 472 (in Chinese).

(严慧, 邱飞, 刀勇, 中国医药工业杂志, 2011, 42(6), 472.)

[8] Sleigh, T.; Savage, D. S.; Mearns, N.; Carlyle, I. G. US 4894369, 1990 [Chem. Abstr. 1989, 110, 115184].

[9] Mendez, J. A.; Mora, M. D.; Rodriguez, A. A. US 7569687, 2009 [Chem. Abstr. 2007, 146, 359039].

[10] Mendes, J. A.; Mora, M. D.; Rodriguez, A. A.; Barragan, E. US 2007/0117975, 2007 [Chem. Abstr. 2007, 146, 359039]

[11] Magni, A.; Grisenti, P. US 6090957, 2000 [Chem. Abstr. 1998, 129, 189525].

[12] Magni, A.; Grisenti, P. US 5808051, 1998 [Chem. Abstr. 1998, 129, 189525].

[13] Wang, H, X. Pharm. Clin. Res. 2012, 212, 135 (in Chinese). (汪会霞, 药学与临床研究, 2012, 212, 135.) 


\title{
辅助材料(Supporting Information)
}

\section{雄甾-3,17-二醇衍生物的分子结构研究}

\author{
方明锋*, $a$ 储 刚 ${ }^{b}$ 李 歆 ${ }^{a}$ 陆国元 ${ }^{*, b, c}$ \\ ( ${ }^{a}$ 南京林业大学化学工程学院 南京 210037) \\ ( ${ }^{b}$ 南京圣和药业公司 南京 210038) \\ (c 南京大学化学化工学院 南京 210093)
}

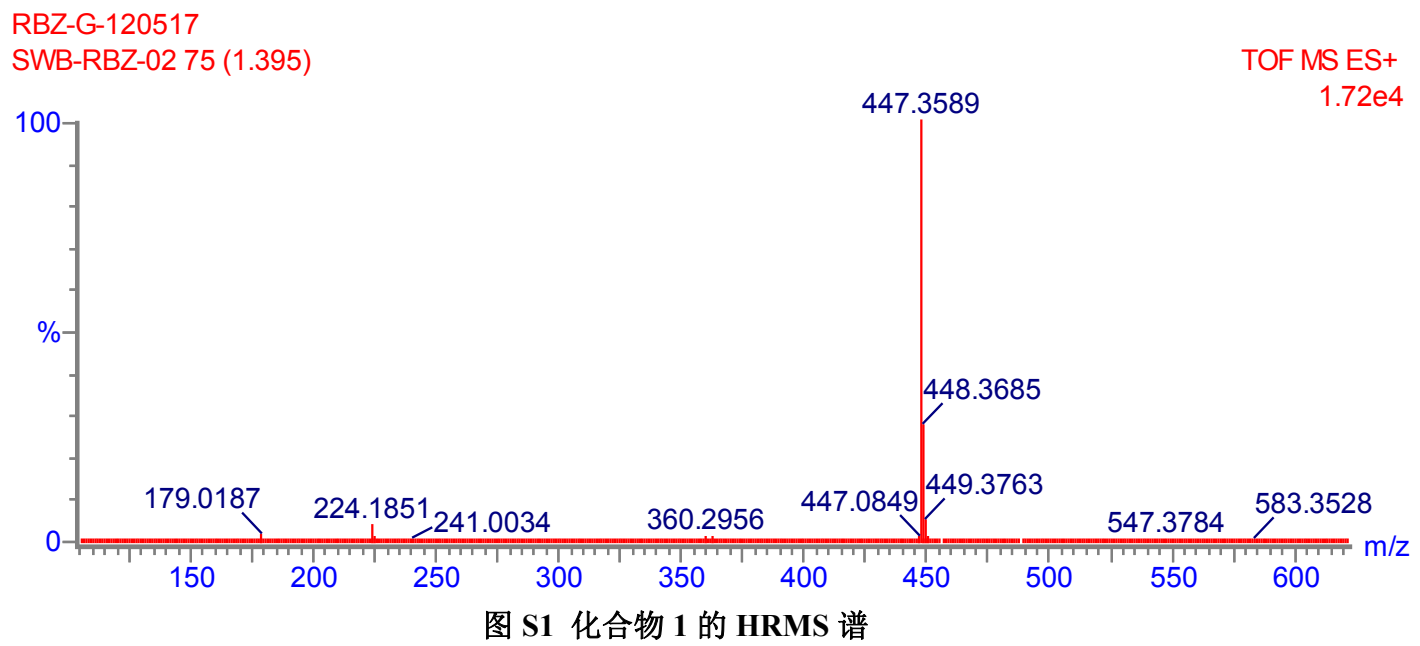

Fig. S1 High resolution ESI-MS for 1 


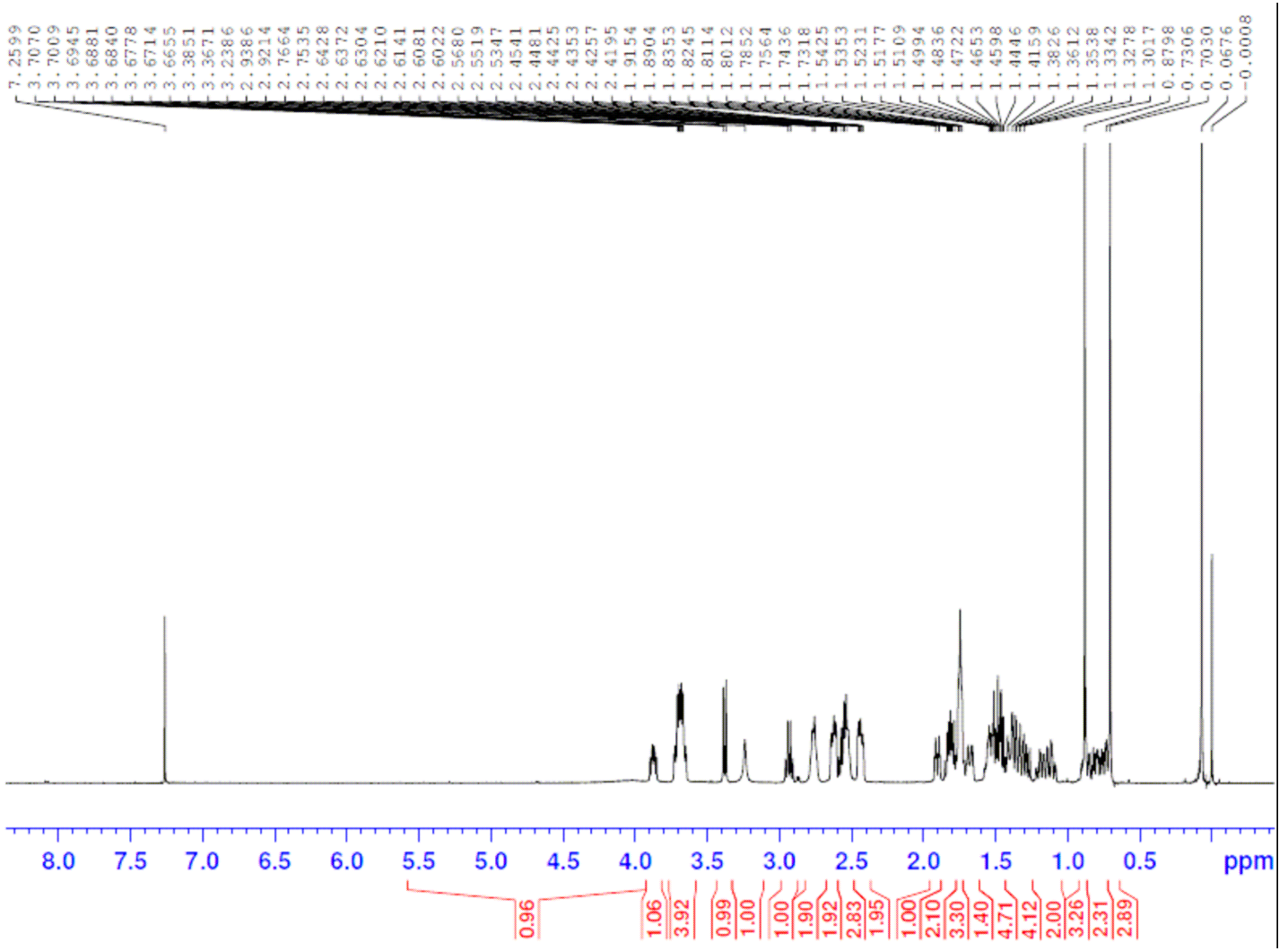

图 S2 化合物 $\mathbf{1}$ 的 ${ }^{1} \mathrm{H} \mathrm{NMR}$ 谱 $\left(500 \mathrm{MHz}, \mathrm{CDCl}_{3}\right)$

Fig. $\mathbf{~ S 2}{ }^{1} \mathrm{H}$ NMR spectrum of $\mathbf{1}\left(500 \mathrm{MHz}, \mathrm{CDCl}_{3}\right)$

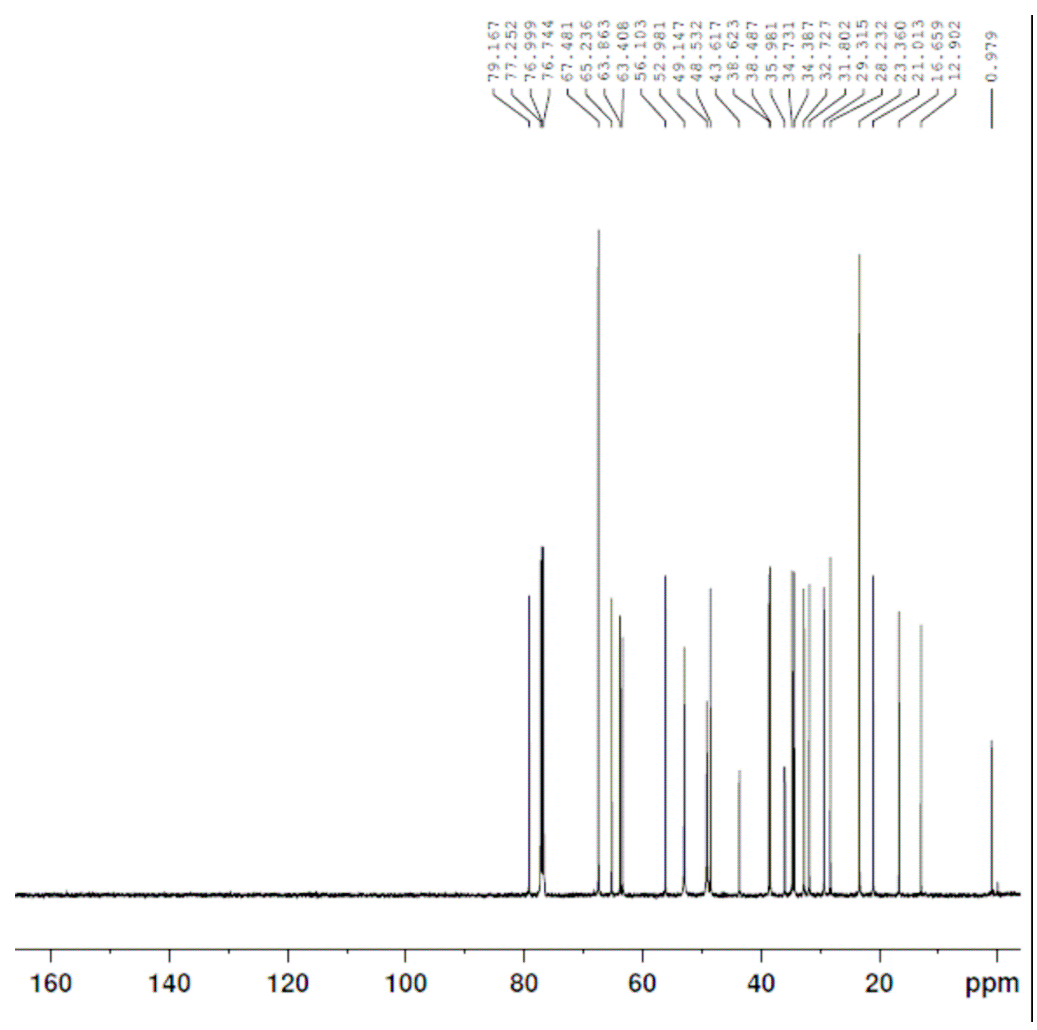

图 S3 化合物 $\mathbf{1}$ 的 ${ }^{13} \mathrm{C} \mathrm{NMR}$ 谱 $\left(125 \mathrm{MHz}, \mathrm{CDCl}_{3}\right)$

Fig. $\mathbf{S 3}{ }^{13} \mathrm{C}$ NMR spectrum of $\mathbf{1}\left(125 \mathrm{MHz}, \mathrm{CDCl}_{3}\right)$ 


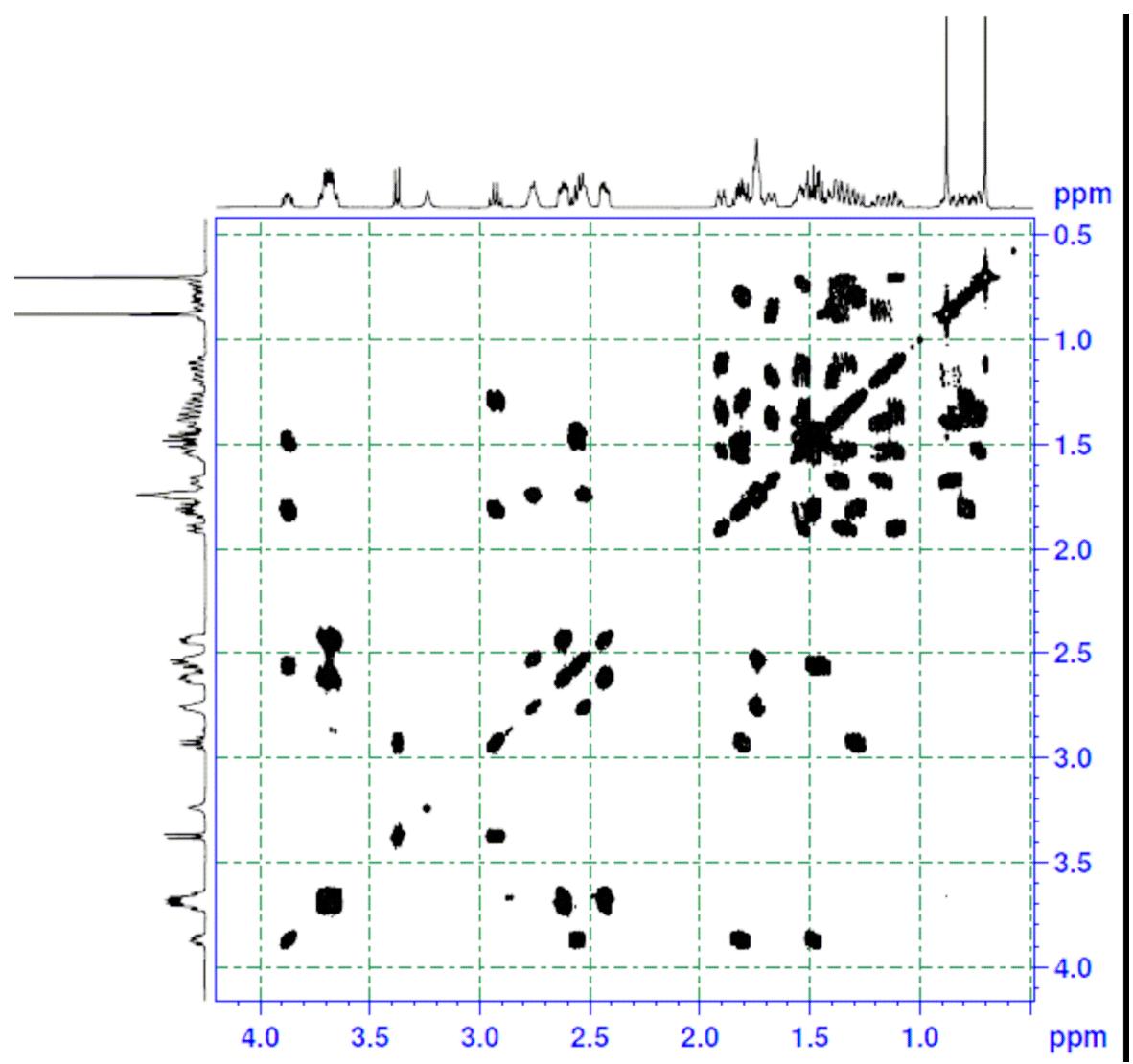

图 S4 化合物 1 的 ${ }^{1} \mathrm{H}-{ }^{1} \mathrm{H}$ COSY 谱

Fig. $\mathbf{S 4}{ }^{1} \mathrm{H}-1 \mathrm{H}$ COSY spectrum of 1

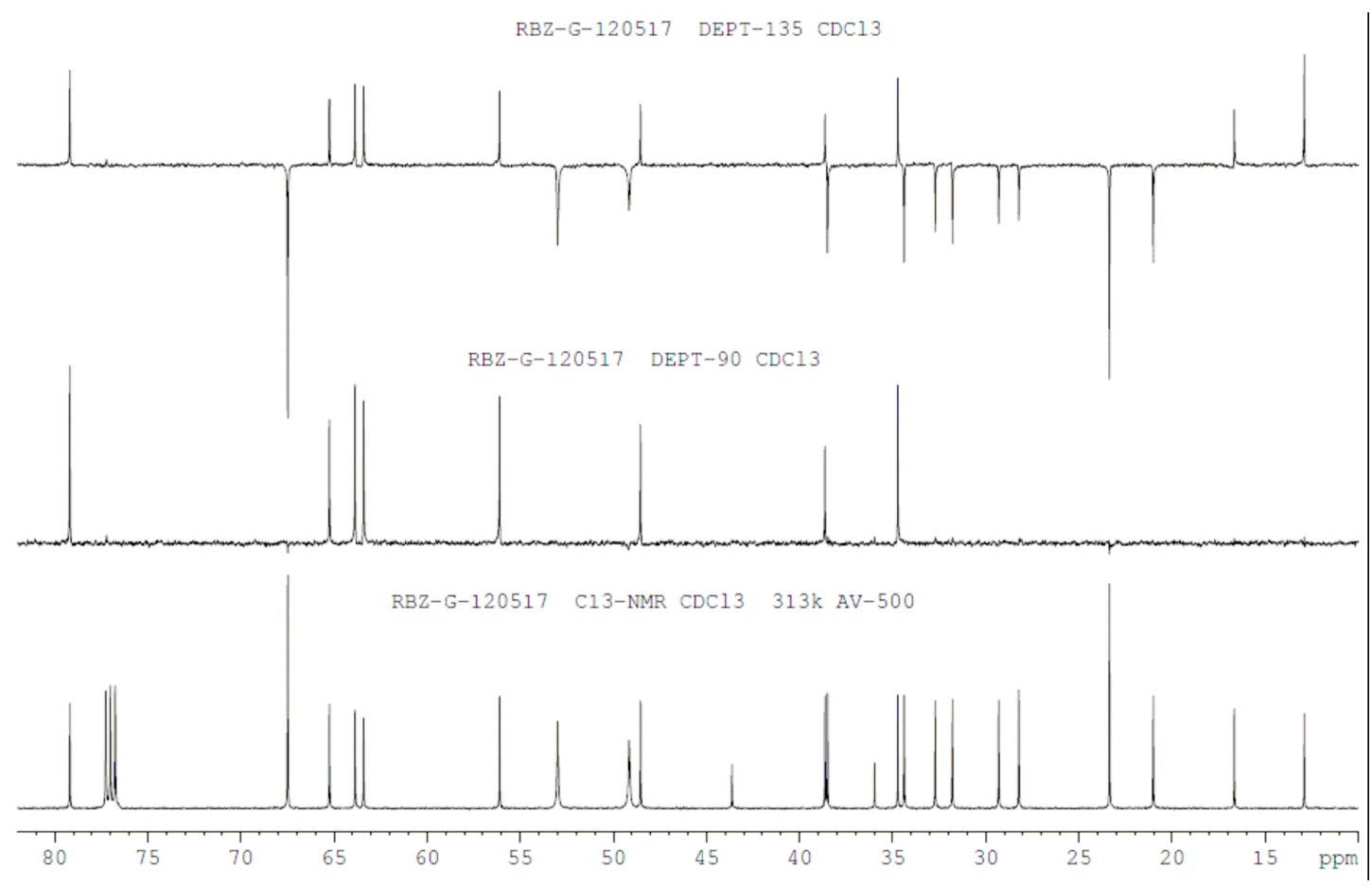

图 S5 化合物 $\mathbf{1}$ 的 DEPT 谱

Fig. S5 DEPT spectrum of $\mathbf{1}$ 


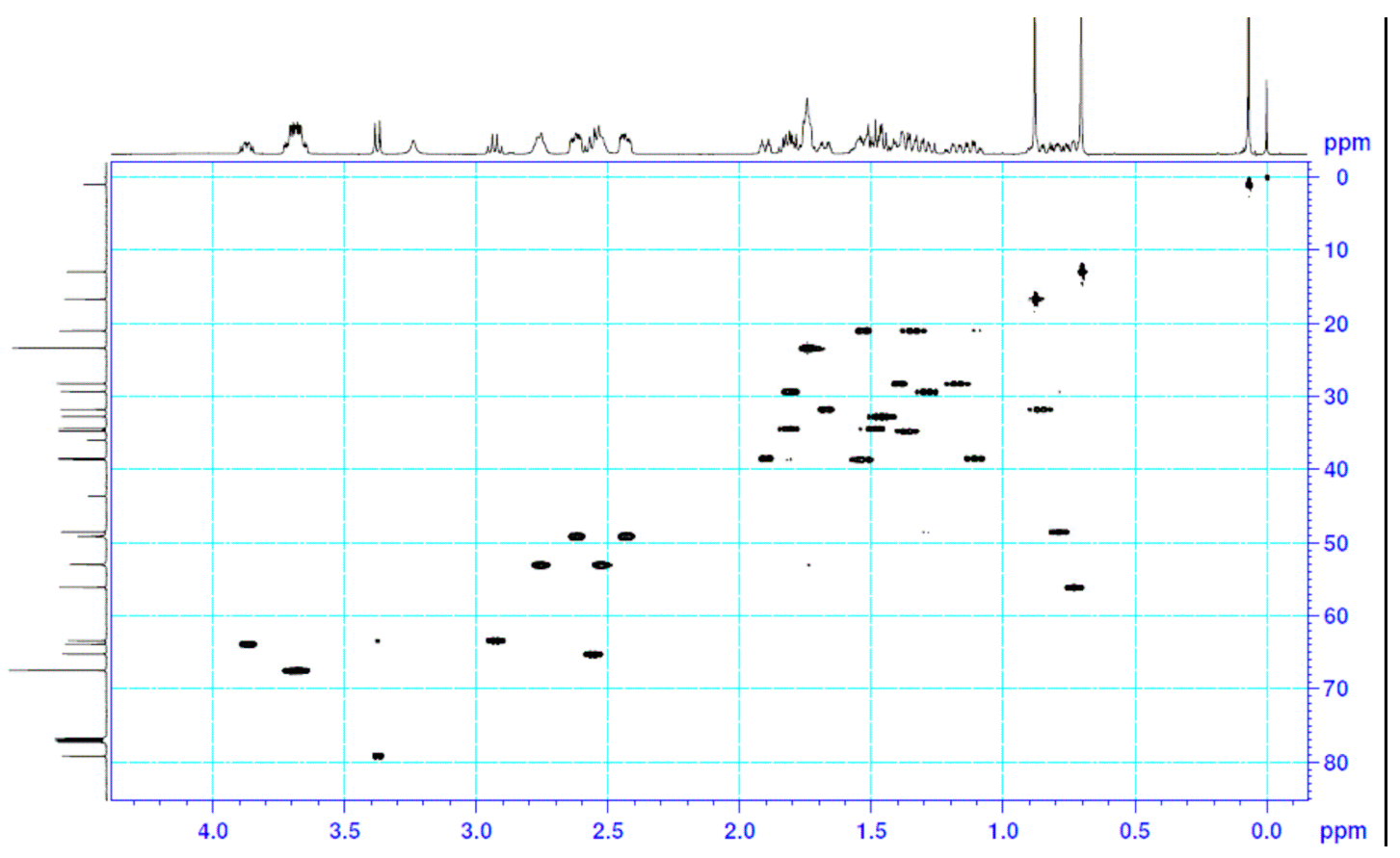

图 S6 化合物 $\mathbf{1}$ 的 HSQC 谱

Fig. S6 HSQC spectrum of $\mathbf{1}$

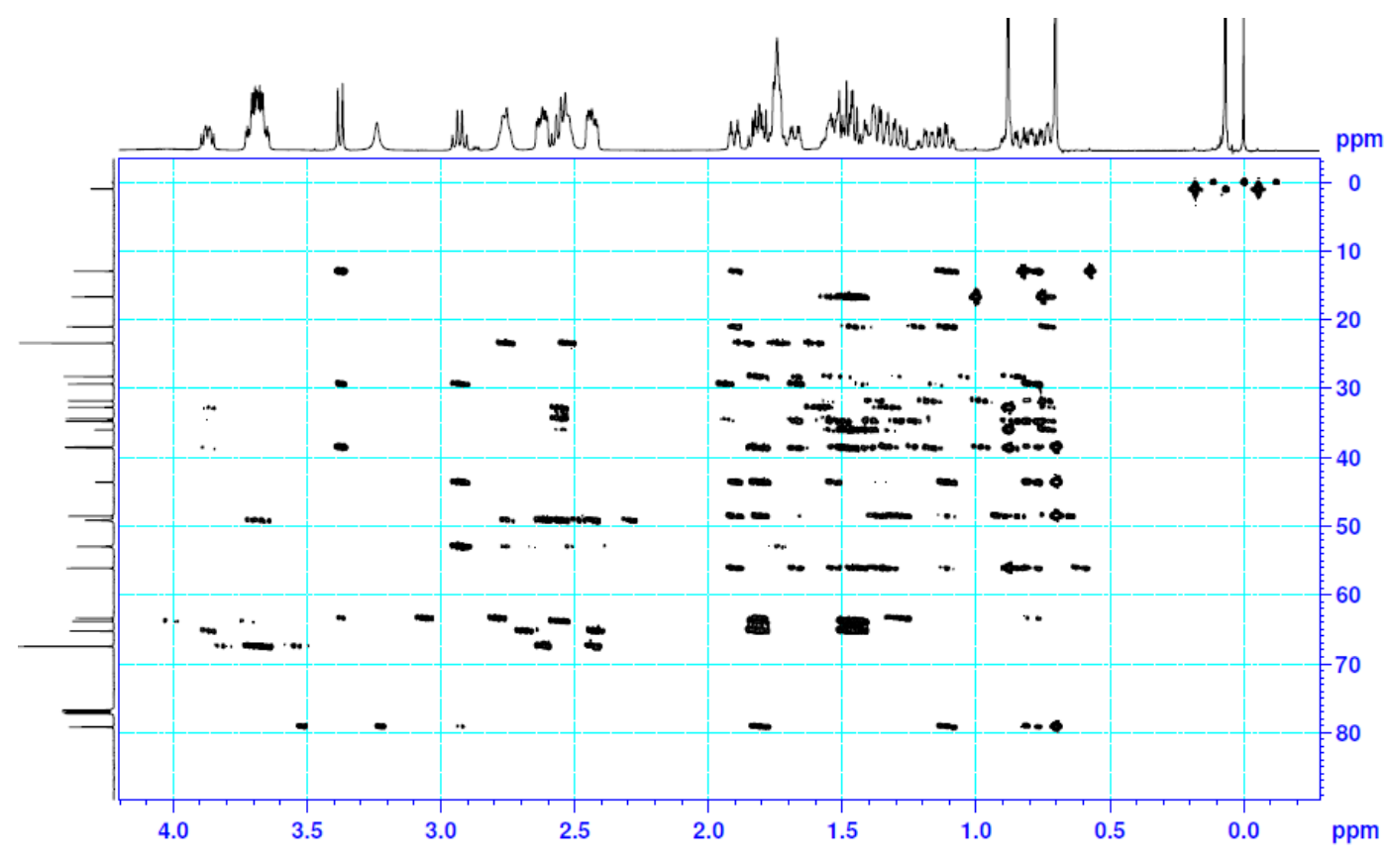

图 S7 化合物 $\mathbf{1}$ 的 HMBC 谱

Fig. S7 HMBC spectrum of $\mathbf{1}$ 


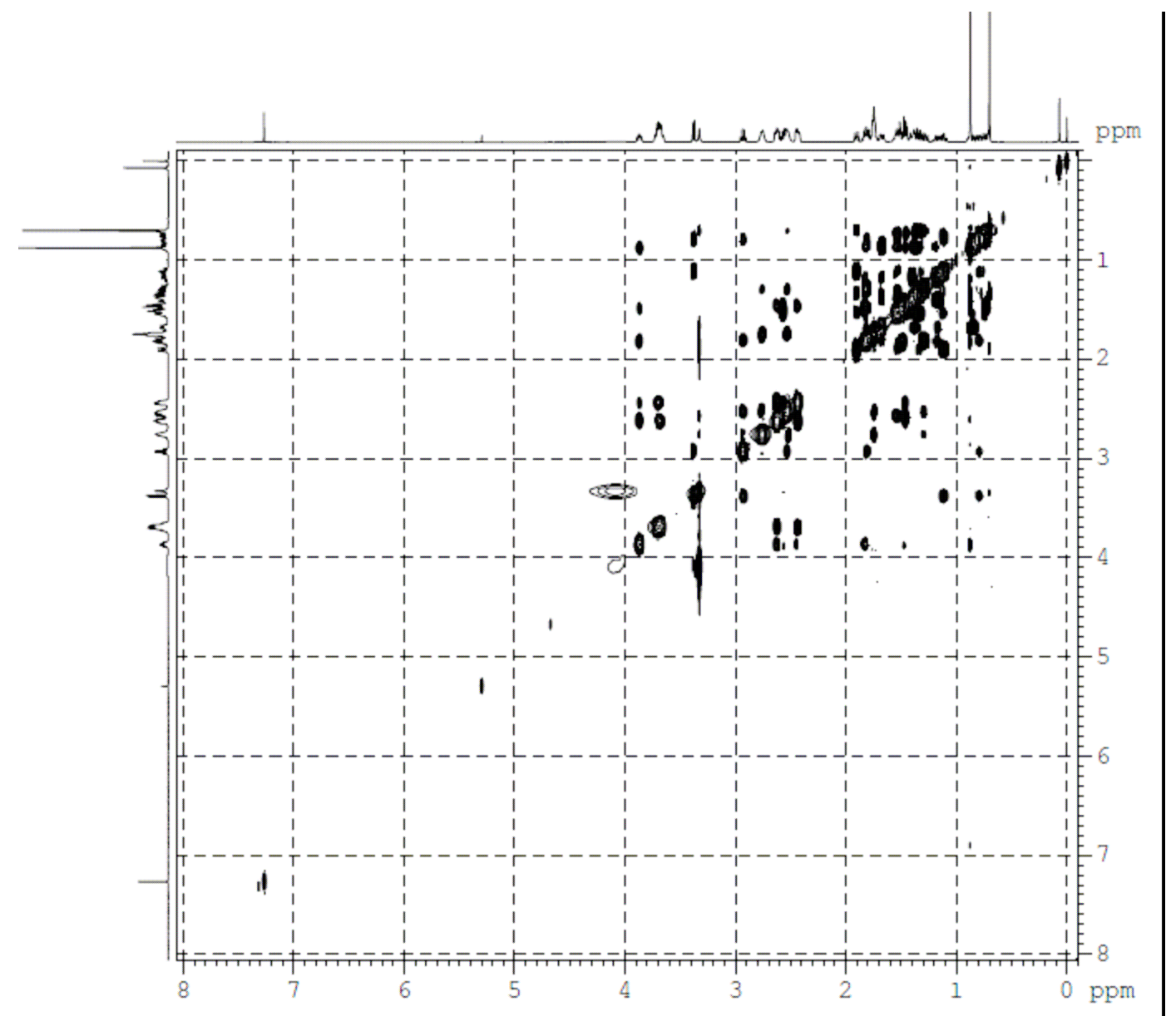

图 S8 化合物 $\mathbf{1}$ 的 NOESY 谱

Fig. S8 NOESY spectrum of 1 